\title{
Intra-abdominal laparoscopic vasectomy by electrocauterization in free range Rhesus macaques (Macaca mulatta)
}

\author{
Vijay Kumar ${ }^{1, ~ *, ~ V i p i n ~ K u m a r ² ~}$ \\ ${ }^{1}$ Veterinary Officer, Monkey Sterilization centre, Gopalpur - Zoo, Palampur, Distt - Kangra, H.P, India \\ ${ }^{2}$ Veterinary Officer, Monkey Sterilization centre, Saster Distt- Hamirpur, Himachal Pradesh, India
}

\section{Email address:}

drvijay0220@gmail.com (V. Kumar)

\section{To cite this article:}

Vijay Kumar, Vipin Kumar. Intra-Abdominal Laparoscopic Vasectomy by Electrocauterization in Free Range Rhesus Macaques (Macaca Mulatta). Animal and Veterinary Sciences. Vol. 1, No. 5, 2013, pp. 42-45. doi: 10.11648/j.avs.20130105.13

\begin{abstract}
The aim of present study was to evaluate intra-abdominal laparoscopic technique in free range male rhesus macaque in terms of rapidity and specificity of the technique. Two hundred twenty free range rhesus macaques were captured by cage trapping method. Anesthesia was achieved using xylazine @ $2 \mathrm{mg} / \mathrm{kg}$ and ketamine @ $10 \mathrm{mg} / \mathrm{kg}$ body weight intramuscularly. One midline and two lateral pre-umblical surgical ports were created. A telescope was inserted through midline port while cutting and grasping forceps were inserted through the lateral ports. About $3-4 \mathrm{~cm}$ long fragment of vas deferens was removed by cutting and cauterization, using atraumatic thermo-cautery forceps and a scissors connected to an electrocautery unit. The induction time, duration of anaesthesia and recovery time was $2.10 \pm 0.20 \mathrm{~min}, 72 \pm 0.28 \mathrm{~min}$ and $38 \pm 0.12 \mathrm{~min}$ respectively. Surgical time for laparoscopic vasectomy was found to be $2 \pm 0.32 \mathrm{~min}$. The method was found to be simple, easy and faster method of vasectomy in the male rhesus macaques.
\end{abstract}

Keywords: Laparoscopic Vasectomy, Pneumoperitoneum, Vas Deferens, Rhesus Macaques (Macaca Mulatta)

\section{Introduction}

Laparoscopic surgery also called as blood less surgery, is a modern technique of surgeries, are now a days used by many surgeon all over the world. Laparoscopic surgery used in various animals, dogs $[1,2,3,4]$ horse [5] and cats $[6,7,8]$ for various surgical interventions. Laparoscopic surgery has a great potential in large scale Animal Birth Control (ABC) programme [9]. Laparoscopic sterilization offers a number of advantages over open methods of sterilizations. It involves minimal invasiveness (keyhole surgery) with maximum visibility, shorter surgical time, decreased postoperative discomfort and pain, less incidence of infection, uncomplicated healing with minimal scarring, no hemorrhages, and no wound dehiscence, and minimal surgical morbidity [11]. Also cost for each procedure and maintenance of equipment is low. In a large scale animal birth control program, the conventional methods of sterilization require a long period between capture and release, due to the time taken for the surgical wounds to heal. In this aspect, keyhole surgery (laparoscopic surgery) has the potential to revolutionize the entire program, as it creates only a very small surgical wound, which usually needs very less postoperative care or regular dressings. Withholding the free range animals for long period from the time of capture to release again in the free range areas may deteriorates the health condition of the animals. Laparoscopic surgery can be an effective method of population control programme at large scale as it requires minimal surgical intervention with less time in performing the surgery and subsequent wound healing is also fast.

The present study was undertaken with the objective to evaluate the distinct advantage of laparoscopic surgical technique in population control programme of rhesus macaques at a large scale in terms of rapidity and specificity of technique with minimal surgical interventions and lesser withholding time of free range rhesus macaques in captivity.

\section{Materials and Methods}

\subsection{Animals}

The present study was carried out on 220 adult male rhesus macaques captured by cage trapping method under Rhesus Macaque population control programme in the State of Himachal Pradesh in India. All the guidelines of animal 
welfare of India regarding the primate's capture and release were followed and the sterilization procedures were carried out after obtaining approval from the Ethical Committee of the Wild Life Wing, Government of Himachal Pradesh, India. The humanely captured animals were transported to Monkey Sterilization Centers in transportation cages and provided adequate feed and water during the transportation. The animals were housed individually in separate cages to avoid stress and infighting injuries.. All the animals were rested for two days with Ad libitum feeding as given above and watering before sterilization. Each animal was given breads 2 no, banana 1 no, black gram $50 \mathrm{gm}$ and cabbage $200 \mathrm{gm}$ as daily feed.

\subsection{Anaesthesia}

The feedand water was withheld about $12 \mathrm{hrs}$ and $2 \mathrm{hrs}$. before anesthesia and surgery, respectively. General anesthesia was administered by intramuscular injection of Ketamine $10 \mathrm{mg} / \mathrm{kg}(100 \mathrm{mg} / \mathrm{ml})$ and Xylazine $2 \mathrm{mg} / \mathrm{kg}$ body weight $(100 \mathrm{mg} / \mathrm{ml})$ (TROY LABORATORIES PTY LTD, Australia). Anesthesia was maintained with ketamine only. Surgical anesthesia was judged to be present if there was an absence of response to cornea reflex and compression of the pelvic limb nail roots.

\subsection{Preparation for Surgery}

Clipping and shaving of the area from post xiphoid up to pubis was done. Asepsis was achieved by scrubbing with $70 \%$ alcohol. Animal was placed in Trendelenburg's (head lower than body) position in dorsal recumbency on the tilted table in Operation Theater.

\subsection{Surgical Procedure}

The animals were securely attached with straps to the surgical table. An incision of $5 \mathrm{~mm}$ was made on preumbilical midline area on the abdominal cavity. A Veres needle connected to $\mathrm{CO} 2$ gas insufflator was carefully inserted through this incision. After insertion of the Veres needle insufflation of the abdominal cavity was achieved by $\mathrm{CO} 2$ gas at the rate of $1 \mathrm{~L} / \mathrm{min}$ with a pressure gradient of 10 $\mathrm{mmHg}$. After induction of pneumoperitoneum the Veres needle was removed and two pre-umbilical paramedian lateral ports were created at 4-5 cm left and right side from the midline port. A $5 \mathrm{~mm}$ safety trocar and cannula was inserted through the first port into the abdominal cavity (Fig. 1). Trocar was taken out and a rigid-type telescope (30 degrees, $5 \mathrm{~mm}$ in diameter, Frontline Co., Germany) connected to a light source (40 W, halogen lamp) was introduced through the cannula (Fig. 2). A monopolar cautery forceps was introduced through the left paramedian port and Grasping forceps inserted through the right paramedian port. The intraperitoneal organs were clearly visualized. The urinary bladder was identified first and then vas deferens and spermatic artery - vein plexus was visualized (Fig. 3). The vas deferens was clearly seen close to the urinary bladder and is visualized by its ivory colored cord like structure. Cautery forceps and monopolar scissors were inserted from bilateral ports to grasp the vas deferens (Fig. 4). A fragment of the vas deferens about 3-4 cm was removed after cutting and thermocauterization, using atraumatic cautery forceps and a scissors connected to an electrocautery unit (Fig. 5). The same procedure was applied for opposite vas deferens.

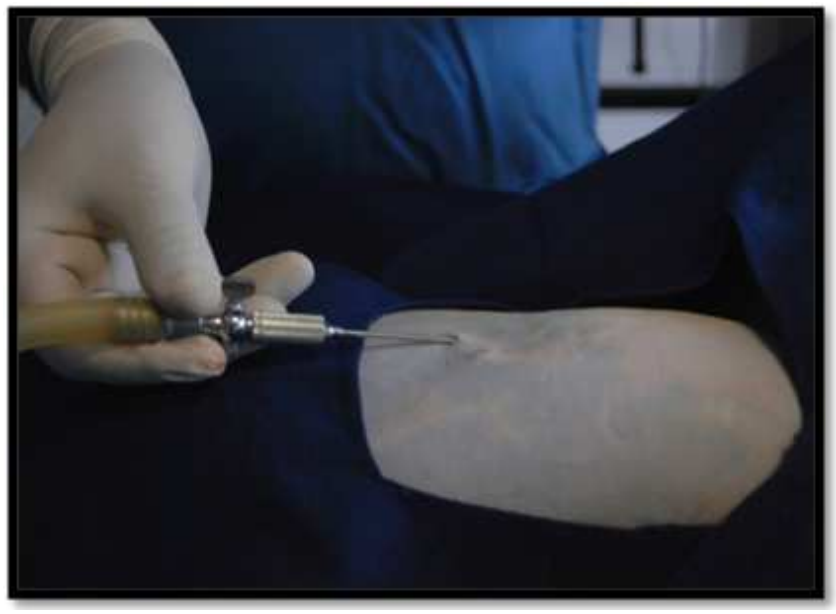

Fig. 1. Pneumoperitoneum by veress needle insertion.

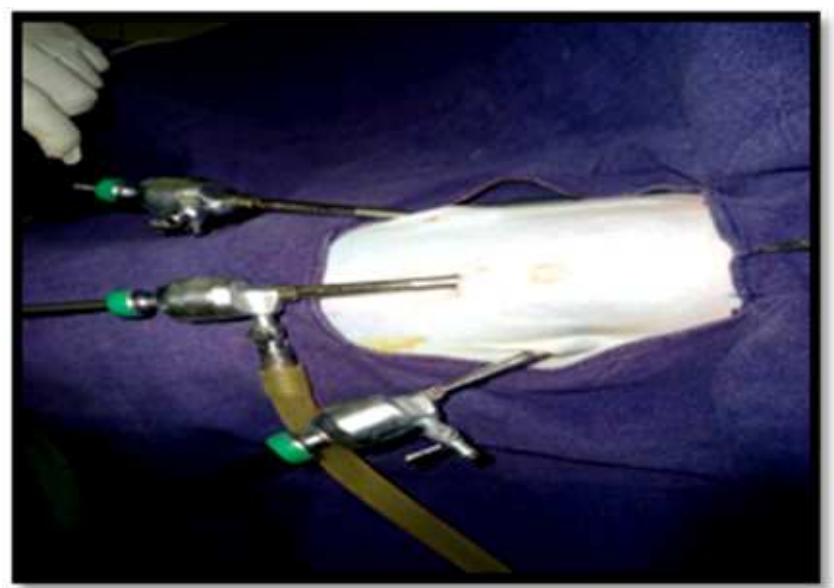

Fig. 2. Insertion of telescope, grasping forcep and monopolar scissor.

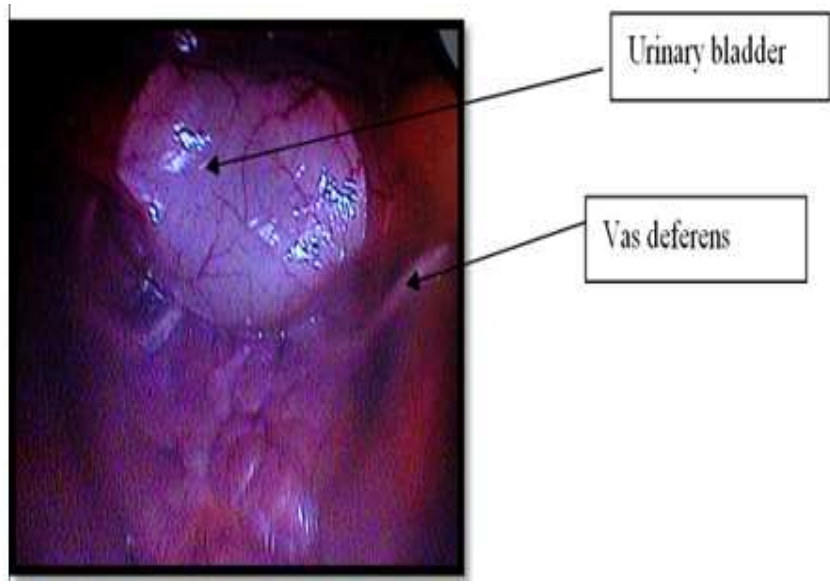

Fig 3. Visualization of internal organs. 


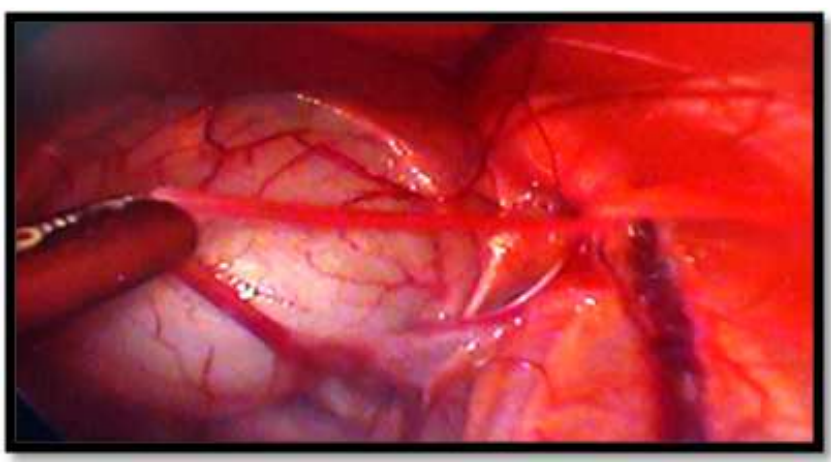

Fig. 4. Grasping of Vas deferens with forcep

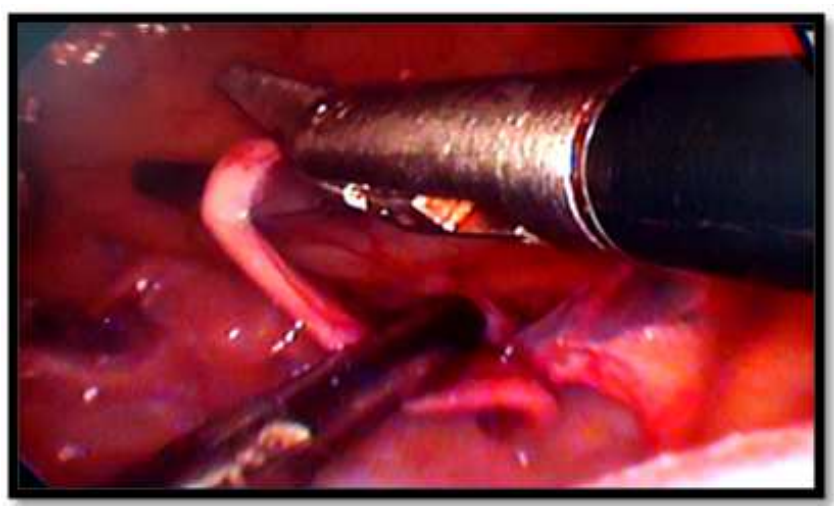

Fig. 5. Cutting and coagulation of Vas deferens.

\section{Results}

Anesthesia for laproscopic vasectomy was performed successfully by using xylazine @ 2mg/kg and ketamine @ $10 \mathrm{mg} / \mathrm{kg}$ body weight. The induction time, duration of anaesthesia and recovery time was found to be $2.10 \pm 0.20$ min, $72 \pm 0.28 \mathrm{~min}$ and $38 \pm 0.12 \mathrm{~min}$ respectively; while mean surgical time for laparoscopic vasectomy was $2 \pm 0.32$ min. The induction of anesthesia was generally calm, smooth with good muscle relaxation throughout the surgical procedure. Of the 220 rhesus macaques only three animals suffered minor bleeding injuries during cut of vas deferens which was checked by electrocauterization of the injured areas. Absorbable surgical suture (Vicryl 3-0, Ethicon) was used to avoid subsequent removal of the stitches on the surgical incisions.

All the rhesus macaques recovered within 6-8 hrs after surgery and started taking feed and water normally. Physiological parameters on an average basis revealed (heart rate $(82 \pm 5 / \mathrm{min})$, respiration rate $(36 \pm 3 / \mathrm{min})$, and rectal temperature $\left(100.8 \pm 0.5^{\circ} \mathrm{C}\right)$ ) normal values. Post operatively, all the animals were given antibiotic and anti-inflammatory coverage viz. Injection Fortivir ( Virbac Animal Health India Private Limited )@10 mg / kg body weight (i/m) and Inj Melonex (Intas Pharmaceuticals Ltd) @ $0.25 \mathrm{mg} / \mathrm{kg}$ body weight $(\mathrm{i} / \mathrm{m})$ for three days. The animals were found fit for release three to five days post surgery with complete surgical wound healing.

\section{Discussion}

Laparoscopic vasectomy has been performed in various animals as well as human [13], however, its use in rhesus macaques is relatively a modern technique. Laparoscopic surgery has shown a significant advancement in the recent years and is indicated in various surgical procedures by the surgeons all over the world. Laparoscopy permitted a clear visualization and identification of the vas deferens. This advantage is due to image amplification and improved illumination in video-surgery. Nevertheless, the conventional technique permitted complete exposition of the vas deferens. The combination of both xylazine and ketamine increases muscle relaxation, recovery time, and duration of anesthesia, while generally decreasing induction time and thermoregulatory ability [4]. To perform laproscopic vasectomy, three trocars were used. Mahalingam et al performed vasectomy in dog with an intra abdominal pressure of $10 \mathrm{mmHg}$. In the present procedure inflation of abdominal cavity was achieved by carbon dioxide gas at $8 \mathrm{~mm}$ of $\mathrm{Hg}$ pressure gradients. The $8 \mathrm{mmHg}$ pressure gradient was sufficient to perform laparoscopic surgery. The $\mathrm{CO}_{2}$ flow rate of $2 \mathrm{~L} / \mathrm{min}$ was also sufficient to maintain intra abdominal pressure during surgery. $\mathrm{CO}_{2}$ is a noninflammable gas and is widely used for use in pneumoperitoneum in laparoscopic procedures. Inflation of abdominal cavity is done in order to get clear visibility and avoid any puncture to internal organs. For creation of pneumoperitoneum, veress needle was used in order to avoid damage to internal organs by directly inserting the trocar and canula intraabdominally. Cauterization of the cut edge of the vas deferens was done and all precautions were taken to avoid any injury to the surrounding organs. A $5 \mathrm{~mm}, 30$ degree oblique angle telescope was found suitable for visualization and to perform vasectomy. There were no major complications except small bleeding during incision and trocar insertion. Longer operative time observed in open methods of vasectomy when compared to laparoscopic vasectomy were consistent with the reported literature in dogs [10] Laparoscopic vasectomy time and complications tend to decrease with an increase in the level of the surgeon's experience. Lesser blood loss in laparoscopic surgery is probably related to lesser tissue damage in the abdominal wall, and better vascular sealing visualization during the electrocoagulation. Intra-abdominal laproscopic vasectomy in llamas and alpacas 2-3 cm sectioning of vas deferens by cautery was described by Bravo and Sumar, (1991) who considered laproscopic vasectomy as a simple, secure and fast technique of vasectomy. The same conclusion was drawn by Silva et al.(1993) in dogs. The procedure was easy, quick and safe. The animals had no complications after surgical intervention and made a good recovery.

\section{Conclusions}

We concluded that Laparoscopic technique permitted clear visualization and identification of abdominal organs 
and vas deferens. The advantages of surgical laparoscopy over the conventional open surgical exploratory laparotomy include faster patient recovery because of smaller surgical sites, improved visualization of abdominal organs, lower postoperative morbidity with lower infection rates, and less postoperative pain and stress. It has proved a boon in animal birth control programme where the extensive surgery is required in minimum time in a large population. The post-surgery recovery is quick and uneventful.

\section{Acknowledgements}

We are grateful to Sh. Sameer Rastogi, Conservator of Forest, North Zone for providing valuable suggestions and help during the population control programme of rhesus macaques. We also thank the team of macaques sterilization centre wild life North Zone for their assistance throughout the work.

\section{References}

[1] Bravo PW and Sumar J. Evaluation of intra-abdominal vasectomy in llamas and alpacas. J Am vet Med Assoc. (1991); 9:1164-1166.

[2] Chariar VM, Shirodkar DN, Khare Y. Minimally invasive technique cuts costs in veterinary surgery.J.Mini Access Surg 2005 April- June.

[3] Dutta A, Maiti S K, Parameswaran A, Kumar N: Evaluation of different laproscopic sterilization techniques in a canine birth control program. Turk J Vet Ani Sc. (2010); 34: 393-402.
[4] Hendrickson DA. History and instrumentation of laparoscopic surgery. Vet Clin N Am-Equine. (2000) ; $16: 233-250$.

[5] Herron MA, Herron M R. Vasectomy in the cat. Mod Vet Pract. (1972) ; 53: 41-43.

[6] Knecht C D. An alternate approach for castration of the dog. Vet Med Small Ani Clini. (1976) ; 71: 469-473.

[7] Mahalingam AN, Kumar SK, Maiti AK, Sharma UD, Kataria M. Laparoscopic sterilization vs. open method sterilization in dogs: a comparison of two techniques Turk J Vet Ani Sc. (2009) ; 33: 427-436.

[8] Naccarato, EF, William SH. Anaesthetic effects of various ratios of ketamine and xylazine in rhesus macaques ( Macaca mulatta). Lab Ani. (1979) ; $13: 317-319$.

[9] Shyu J J, Liu PC. Castration of male dogs using laparoscopic electro-coagulation. J Chin Soc Vet Sci. (1998) ; 24: 169-178.

[10] Silva LD, Onclin K, Donnay I, Verstegen JP : Laproscopic vasectomy in the male dog. J Rep. and Fert. Suppl. (1993); 47: 399-401.

[11] Veenis M. Sterilizing female dogs. Can Vet. J. (2004) ; 45 : 347-8.

[12] Wildt DE, Lawler DF. Laparoscopic sterilization of the bitch and queen by uterine horn occlusion. Am J Vet Res. (1985) : 46: 864-869.

[13] Wildt DE, Seager CH, Bridges. Sterilization of male dog and cat by laparoscopic occlusion of S.W.J. the ductus deferens. Am J Vet Res. (1981) ; 42: 1888-1897. 